5 KRUG. P.A., STOLTE, R., and ULRICH, R.: 'Measurement of index modulation along an optical fiber Bragg grating', Opt. Lett., 1995, 20, (17), pp. 1767-1769

6 DYER, P.E., FARLEY, R.J., GIEDL, R., and BYRON, K.C.: 'Amplification of fibre Bragg grating reflectivity by post-writing exposure with a 193 nm ArF laser', Electron. Lett., 1994, 30, (14), pp. 1133-1134

7 NIAY, P., BERNAGE, P., LEGOUBIN, S., DOUAY, M., BAYON, J.F., GEORGES, T., MONERIE, M., and POUMELLEC, B.: 'Behaviour of spectral transmissions of Bragg gratings written in germania-doped fibres : Writing and erasing experiments using pulsed or $\mathrm{CW}$ uv exposure', Opt. Commun., 1994, 113, pp. 176-192

\section{Linewidth broadening of Er-doped superfluorescent fibre source using long- period grating}

\section{C.D. Su and L.A. Wang}

It is demonstrated that, by using a long period grating, a doublepass backward superfluorescent fibre source obtains a broader linewidth over a large pump region while still maintaining a comparable output power compared to that without the grating.

Introduction: Rare-earth-doped superfluorescent fibre sources (SFSs) have been used for low-coherence sensor applications. To reduce resolution deterioration due to coherent effects, a source with broad linewidth is required. Several methods have been used to broaden the amplified spontaneous emission (ASE) spectrum $[1-5]$. These techniques share a common principle: the addition of a filter to induce higher spectral losses in the high-intensity region so that the ASE spectrum is reshaped. Blazed gratings have been used to broaden the ASE spectra $[1,2]$; however, the output power decreased markedly when the filters were added at the output end of the ASE sources. In [3], a long period grating (LPG) was added between two erbium-doped fibres to redistribute the gain in a single-pass backward (SPB) configuration, and the excess loss was $\sim 0.5 \mathrm{~dB}$. It has been reported that a double-pass backward (DPB) SFS would have a broader linewidth and better pump efficiency compared to an SPB one [6]. As for the ways of increasing the linewidth of a DPB SFS, an array of screws serving as attenuator with a high reflectance mirror [4] and multiple Bragg gratings as mirror [5] have been demonstrated. In this Letter, we demonstrate a new technique by adding a conventional long period grating in a DPB configuration for linewidth increase. Such a technique is simpler than those reported before in the same DFB configuration, and is shown to have a broader linewidth than and comparable output power to a DPB SFS without any grating.

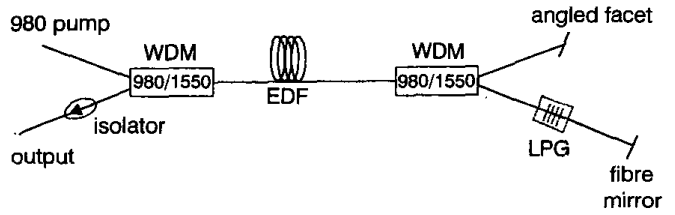

73211

Fig. 1 Schematic diagram of DPB SFS configuration with or without $L P G$

Experiment setup: Fig. 1 shows the setup of an Er-doped SFS in DPB configuration both with and without an LPG. The output power from a laser diode operating at $976 \mathrm{~nm}$ wavelength is launched through a wavelength division multiplexer (WDM) into a $20 \mathrm{~m}$ long Er-doped fibre (FIBERCORE DF1500F-980). The residual pump power is separated by a second WDM, and the forward ASE is filtered by a wavelength-dependent reflector, which is composed of an LPG and a fibre mirror. An isolator with $\sim-60 \mathrm{~dB}$ isolation is used to prevent the formation of a resonant cavity due to the optical feedback from the output port. The LPG is externally written in a hydrogenated dispersion shifted fibre by using a $\mathrm{KrF}$ excimer laser. After exposure, a periodic index variation is formed in the core region with period of $400 \mu \mathrm{m}$. The amount of index variation is a function of the exposure intensity and time.
The guided mode is coupled into the forward cladding modes by the imprinted LPG when the phase matching condition is satisfied [7]. The cladding modes disappear after propagating a distance due to weak confinement, resulting in several loss bands whose peak wavelengths, bandwidths, and peak losses are controllable. An LPG with appropriate spectral characteristics is chosen to induce an excess loss in the dominant band or bands of the original DPB SFS (the one without an LPG) to redistribute the spectral power for linewidth broadening.

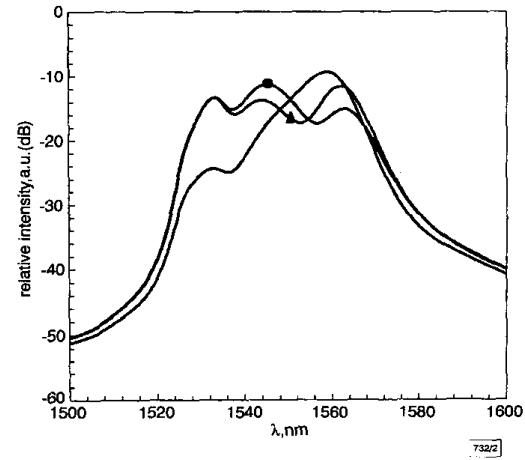

Fig. 2 Output spectra of DPB SFSs

DPB

DPB with LPG

DPB with LPG2

Results: Fig. 2 shows the ASE spectra of DPB SFSs pumped at $85 \mathrm{~mW}$ with and without the presence of an LPG. The original Erdoped DPB SFS has two major bands, one centred at $\sim 1532 \mathrm{~nm}$ and the other at $\sim 1558 \mathrm{~nm}$, and the spectrum evolution is affected by several parameters such as pump power, EDF length etc. The ASE spectrum of the DPB SFS without an LPG shown in Fig. 2 represents an extreme case, which is dominated by the $\sim 1558 \mathrm{~nm}$ band. The linewidth of the ASE spectrum as defined in [8] is $\sim 21 \mathrm{~nm}$, or equivalently $\sim 11 \mathrm{~nm}$ for FWHM. To enable the reshaping of such an output spectrum that has only one dominant band requires the loss spectrum of an LPG peak near the dominant band. Two LPGs, LPG1 and LPG2, with different characteristics are used to determine the effect of spectrum reshaping. LPG1 and LPG2 have peak losses of 8.9 and $8.6 \mathrm{~dB}$ at peak wavelengths of 1558 and $1561 \mathrm{~nm}$, and have FWHMs of 13.5 and $12 \mathrm{~nm}$, respectively. For the DPB SFS with either LPG, the forward ASE signal, the spectral components of which are not within the loss band of the LPG, is completely reflected, and passes through the EDF twice. Conversely, the ASE signal within the loss band is coupled into cladding modes and is partially reflected. Since there is less stimulated emission in the spectral range of the LPG's loss band, the gain is redistributed and the output ASE spectrum is broadened as shown in Fig. 2. The insertion of the LPG causes the intensity of the $1558 \mathrm{~nm}$ band to decrease and that of the $1532 \mathrm{~nm}$ band to increase. It is noted that the original $1558 \mathrm{~nm}$ band disappears and two additional bands centred at $\sim 1544$ and $\sim 1562 \mathrm{~nm}$ are generated. The linewidth of the output ASE increases from $\sim 21 \mathrm{~nm}$ for the DPB SFS without an LPG to $\sim 36$ and $\sim 39 \mathrm{~nm}$ with LPG1 and LPG2, respectively. As observed from the spectra shown in Fig. 2, the intensity of the $\sim 1544 \mathrm{~nm}$ band is smaller than that of the $\sim 1562 \mathrm{~nm}$ band when LPG1 is used and is larger when LPG2 is used. Although the relative intensity of the two bands is affected by the location of the peak loss wavelength of the LPG, the resultant linewidth broadening is clearly seen for both LPGs. To further reduce the ripples of the ASE spectrum, an LPG with peak loss wavelength(s) closer to the peak of major band(s) of the original DPB SFS is needed, and is under study.

Fig. 3 shows the output power against the pump power for the DPB SFSs. Since the forward ASE is weak, the loss caused by the mode coupling of an LPG is small. Therefore, the threshold pump power, the output power, and the pump efficiency suffer little degradation when the LPG is used. The efficiency is $\sim 50 \%$ after correcting the insertion losses of the input/output WDM $(\sim 0.6 \mathrm{~dB})$ and the isolator $(\sim 0.9 \mathrm{~dB})$. The efficiency and the linewidth are larger than that reported in [5], which requires the use of either 
multiple Bragg gratings or a specialised fibre grating instead of only one LPG as shown in our case.

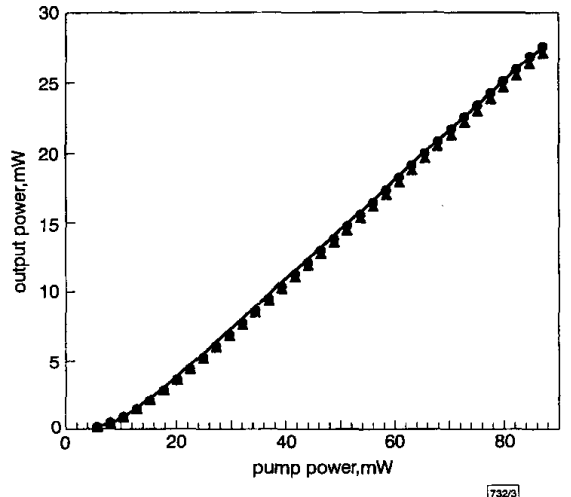

Fig. 3 Output power against pump power

DPB

DPB with LPG1

DPB with LPG2

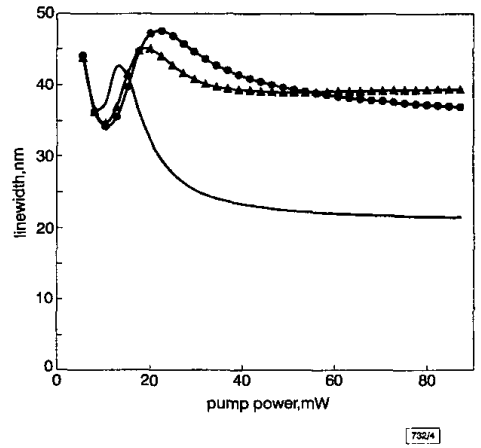

Fig. 4 Linewidth against pump power

A DPB with LPG1

DPB with LPG2

Fig. 4 shows the dependence of linewidth on pump power. For pump powers larger than $40 \mathrm{~mW}$, the linewidth for all DPB SFSs decreases slowly as the pump power increases. The linewidth remains nearly constant, $\sim 39 \mathrm{~nm}$, for pump powers ranging from 40 to $85 \mathrm{~mW}$ for the DPB SFS with LPG1.

The major advantage of this technique is that a broad linewidth can be obtained over a large pump power range with little power degradation. It can be shown to be effective also for DPB SFSs with two dominant bands by using two LPGs each with corresponding loss bands or one LPG with two adjacent loss bands for spectral power redistribution.

Conclusion: We have demonstrated that, by inserting an appropriate long period grating in a DPB SFS, the linewidth can be increased almost as much as twofold over a large pump power region with comparable output power and pump efficiency as compared to that without a grating.

(C) IEE 1999

Electronics Letters Online No: 19990173

24 November 1998 DOI: 10.1049/el:19990173

C.D. Su and L.A. Wang (Institute of Electro-Optical Engineering, National Taiwan University, Taipei, Taiwan, Republic of China)

L.A. Wang: Corresponding author

E-mail: lon@ccms.ntu.edu.tw

\section{References}

1 Wilkinson, M., BebBington, A., CASSIDY, S.A., and MCKEe, P.: 'Dfiber filter for erbium gain spectrum flattening', Electron. Lett., 1992, 28, pp. 131-132

2 KASHYAP, R, WYATT, $R$, and CAMPBELL, R.J.' 'Wideband gain flattened erbium fiber amplifier using a photosensitive fiber blazed grating', Electron. Lett., 1993, 29, pp. 154-156
3 HOdGSON, C.W., and venGSARKAR, A.M.: 'Spectrally shaped highpower amplified spontaneous emission sources incorporating longperiod gratings'. Optical Fiber Communication Conf. Tech. Dig., 1996, Paper TuG3

4 PASCHOTTA, R., NILSSON, J., TROPPER, A.C., and HANNA, D.C.: 'Efficient superfluorescent light source with broad bandwidth', IEEE J. Sel. Topics Ouantum Electron., 1997, 3, pp. 1097-1099

5 DYER, S.D., and ROCHFORD, K.B.: 'Spectral tailoring of erbium superfluorescent fiber source', Electron. Lett., 1998, 34, pp. 1137 1139

6 WANG, L.A., and CHEN, C.D.: 'Stable and broadband Er-doped superfluorescent fiber sources using double-pass backward configuration', Electron. Lett., 1996, 32, pp. 1815-1817

7 VENGSARKAR, A.M., LEMAIRE, P.J., JUDKINS, J.B., BHATIA, V. ERDOGAN, T., and SIPE, J.E.: 'Long-period fiber gratings as bandrejection filters', J. Lightwave Technol., 1996, 14, pp. 58-65

8 HALL, D.C., BURNS, W.K, and MOELLER, R.P.: 'High-stability $\mathrm{Er}^{3+}$ doped superfluorescent fiber sources', J. Lightwave Technol., 1995, 13, pp. $1452-1460$

\section{Low birefringence measurement in optical fibres}

E.A. Kuzin, B. Ibarra Escamilla and J.M. Estudillo Ayala

A new method for measuring low birefringence in optical fibres is described. It is based on a fibre twist in a fibre optical loop mirror. It allows birefringence measurement within the range $10^{-6}$ $-0.5 \times 10^{-8}$. Neither specific input polarisation nor previous principal fibre axes orientation adjustment are required.

Most experimental techniques proposed for measuring fibre birefringence use a section of fibre several beat-lengths long [1]. These techniques are convenient for hi-birefringent fibre, but they are difficult to use for low-birefringence fibres. A recent proposal was to use the twist method for low-birefringence fibres [2]. In this technique a fibre section is placed between crossed polarisers oriented parallel to the principal axes of the fibre. The fibre is then twisted and the transmission characteristics of this system measured. This technique provides high accuracy when measuring fibres shorter than one beat length, but the procedure for adjusting the polarisers with respect to the principal axes of the fibre is very time consuming, which restricts its practical applications.

A fibre optical loop mirror (FOLM) can operate as a polarimetric type device with unpolarised input light and does not require any previous adjustment of the principal axes of the fibre with respect to the laboratory co-ordinate system $[3,4]$.
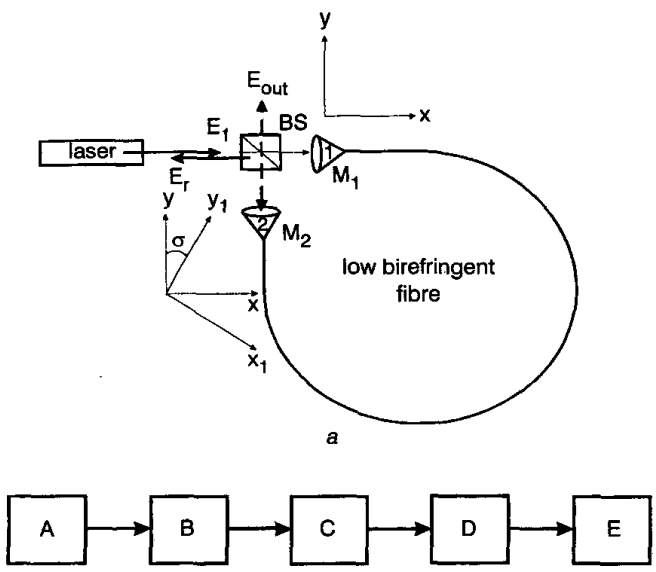

$b$

$6 \longdiv { 6 1 8 1 1 }$

Fig. 1 Schematic diagram of FOLM used in experiments

Here we show that an FOLM can be used for very simple measurement of the birefringence of low-birefringence fibres. In the experiment (Fig. 1), the laser beam from a CW Nd:YAG laser was 